\title{
L'école virtuelle : faute de pain, on mange de la galette!
}

\section{Virtual school: for lack of bread, we eat pancakes!}

\section{La escuela virtual: ¡a falta de pan, buenas son tortas!}

https://doi.org/10.52358/mm.vi8.201

Isabelle Carignan, professeure

Université TÉLUQ, Canada

isabelle.carignan@teluq.ca

Steve Bissonnette, professeur

Université TÉLUQ, Canada

steve.bissonnette@teluq.ca

Marie-Christine Beaudry, professeure

Université du Québec à Montréal, Canada

beaudry.marie-christine@uqam.ca

\section{RÉSUMÉ}

École à distance, cours asynchrone, cours en ligne synchrone : la pandémie mondiale entraine une scolarisation des élèves à la fois en présentiel et en virtuel. Le présent article propose une réflexion sur l'école virtuelle. Basé à la fois sur des observations de parentschercheurs et des écrits scientifiques, l'article met en relief les points positifs et négatifs liés à l'école virtuelle. II ressort notamment de cela que l'école virtuelle ne peut devenir une solution à long terme, et ce, ni pour les élèves et leurs enseignants, ni pour les parents. L'école virtuelle constitue donc une solution d'urgence à court terme pendant la pandémie.

Mots-clés : école virtuelle, enseignement à distance, avantages, inconvénients, conciliation/superposition école-famille-travail 
Distance school, asynchronous course, synchronous online courses: the global pandemic is leading to students' schooling both face-to-face and virtual. This article offers a reflection on the virtual school. Based on both parent-researcher observations and scientific literature, the article highlights the positives and negatives of virtual school. In particular, the virtual school can become a long-term solution, neither for the pupils and their teachers nor for the parents. The virtual school is, therefore, a short-term emergency solution during this pandemic.

Keywords: virtual school, distance learning, advantages, disadvantages, school-familywork puzzle

RESUMEN

Escuela a distancia, curso asincrónico, curso en línea sincrónico: la pandemia global está propiciando la escolarización de los estudiantes tanto presencial como virtualmente. Este artículo ofrece una reflexión sobre la escuela virtual. Basado en observaciones de padres e investigadores y literatura científica, el artículo destaca los aspectos positivos y negativos de la escuela virtual. En particular, resulta que la escuela virtual no puede convertirse en una solución a largo plazo, ni para los alumnos y sus profesores, ni para los padres. La escuela virtual es, por tanto, una solución de emergencia a corto plazo durante la pandemia.

Palabras clave: escuela virtual, educación a distancia, ventajas, desventajas, rompecabezas escuela-familia-trabajo

La pandémie mondiale de COVID-19 a mené à des confinements, à des déconfinements et à des (re)(dé)confinements qui ont entrainé leur lot de cours à distance pour les enfants. Les parents ont alors dû jongler avec leur vie professionnelle et leur vie familiale pour gérer et organiser l'enseignement virtuel de leurs enfants. Voici un article de réflexion sur ce sujet d'actualité qu'est l'école virtuelle. II sera donc question des points positifs et négatifs liés à l'école virtuelle, dégagés à la fois d'observations faites par des parents - dont deux des auteures du présent article - et de conversations par courriel et en visioconférence avec des enseignants d'expérience. Un parallèle sera fait avec les écrits scientifiques récents dans le domaine. Ce qui ressort notamment de ces observations est le fait que l'école virtuelle ne peut devenir une solution à long terme, ni pour les élèves et leurs parents, ni pour les enseignants : la recension de recherches scientifiques montre d'ailleurs les effets négatifs de ce type d'école sur le rendement des élèves. Par conséquent, l'école virtuelle est une solution d'urgence à mettre en place à court terme, puisque l'effet enseignant est « dilué » et qu'il est difficile, voire impossible pour les parents, de concilier l'école virtuelle en mode synchrone et le travail, particulièrement avec de jeunes enfants. 


\section{L'effet enseignant « dilué »}

L'effet enseignant est l'influence qu'un enseignant peut avoir sur ses élèves. Le chercheur John Hattie est celui qui est le plus connu relativement à ce concept; il a analysé plus de 800 méta-analyses à ce sujet (Hattie, 2008). Tout en soulignant les nombreuses critiques associées aux études de ce chercheur, Bissonnette et Boyer (2019), à l'instar de William (2018), ont montré l'importance de l'effet enseignant à la lumière des recherches menées sur les « caractéristiques qualitatives des enseignants " (teacher quality). Dans ce type de recherche, l'effet enseignant est révélé en comparant les progrès des élèves entre le début et la fin d'une année scolaire, à l'aide d'une épreuve standardisée.

Aaronson, Barrow et Sander (2007) analysent les caractéristiques qualitatives d'enseignants de $9^{\mathrm{e}}$ année dans les écoles publiques de Chicago sur la base des progrès de leurs élèves au cours d'une année scolaire. Sur cette base, les enseignants ont été divisés en trois catégories : très efficaces, moyennement efficaces et peu efficaces. Or, les élèves assignés aux enseignants très efficaces font $40 \%$ plus de progrès sur une année scolaire que ceux confiés aux enseignants moyennement efficaces tandis que les élèves assignés aux enseignants peu efficaces font $30 \%$ moins de progrès que ceux confiés aux enseignants moyennement efficaces. En d'autres mots, le progrès des élèves assignés aux enseignants très efficaces est deux fois plus élevé que celui des élèves confiés aux enseignants peu efficaces. (Bissonnette et Boyer, 2019, p. 116)

Ainsi, un facteur qui a un impact positif sur la réussite des élèves est, sans contredit, l'enseignant. Celuici peut avoir un effet positif sur l'apprentissage des élèves s'il apporte du soutien à ses élèves, si la relation est positive, si les rôles sont bien établis du côté de l'enseignant et de l'élève, et si les stratégies pédagogiques (Messier, 2019) sont ajustées, au besoin, pour favoriser l'apprentissage et la réussite des élèves.

Mais qu'en est-il de l'effet enseignant lors d'un enseignement virtuel en mode synchrone? II est indéniable que l'effet enseignant n'est pas le même à l'écran. En effet, le fait d'être devant l'écran « déshumanise » en quelque sorte le contact social :

se voir en mode [synchrone] parfois dégradé (dans de petites fenêtres vidéos dont le flux peut être perturbé), la distance physique, l'immobilité des participants et les difficultés à interagir dans une classe à effectif important peuvent mettre à mal la dynamique de groupe (Tellier, 2020, p. 3).

Certains enseignants nous ont confié être mal à l'aise devant l'écran, car ils se sentent limités dans leurs mouvements et dans les stratégies d'enseignement-apprentissage qu'ils mettent en œuvre. D'autres ont mentionné aimer que les parents soient à côté de l'enfant pour lui venir en aide, mais ont aussi soulevé le fait que la présence des parents entraine son lot de défis : parents qui donnent la réponse à l'enfant, qui ouvrent le micro pour l'enfant alors que l'enseignant souhaite justement apprendre aux enfants comment ouvrir et fermer le micro, parents qui commentent les propos des autres enfants... Du côté des élèves, certains enfants sont très stressés à l'idée de prendre la parole à l'écran devant leur groupe (Carignan, Beaudry et Cohene, 2020) et cet état peut jouer sur leur sentiment d'efficacité personnelle (Bandura, 2013). En effet, certains enfants habituellement confiants en salle de classe n'arrivent plus à réaliser les tâches demandées : ils ont l'impression que tous les regards sont braqués sur eux, que les erreurs seront plus visibles face au groupe, et ce, même si l'enseignant met tout en place pour que les enfants se sentent à l'aise. L'enseignant fait bien sûr tout ce qu'il peut pour être intéressant, pour attirer et garder l'attention des enfants, pour diversifier son enseignementapprentissage, mais, particulièrement avec les problèmes techniques (problème de son, d'images, etc.), les élèves ne sont pas toujours concentrés sur ce que l'enseignant fait. 
Néanmoins, trois points positifs semblent émerger de l'école virtuelle en mode synchrone, en temps de pandémie : 1) les enfants sont contents de voir leur enseignant et leurs amis à l'écran; 2) la classe virtuelle permet aux parents d'avoir une idée de ce qui est enseigné comme notions en salle de classe et la façon dont le tout est enseigné; 3) l'enseignant peut donner de la rétroaction en temps réel, si le temps le permet. La rétroaction positive des enseignants et des parents est primordiale lors de l'enseignement virtuel en mode synchrone pour que les élèves restent motivés (Baudoin, Dellisse, Lafontaine, Coertjens, Crépin, Baye et Galand, 2020), persévèrent et se sentent compétents (Bandura, 2013). Toutefois, la rétroaction et la gestion de classe virtuelles peuvent devenir de grands défis à relever lorsque le groupe excède une dizaine d'élèves à l'écran.

\section{La gestion de classe virtuelle}

Sans contredit, la gestion de classe virtuelle peut devenir problématique (Lee et Gage, 2020) et se doit d'être adaptée au contexte de pandémie (Caron, Gaudreau, Harvey, Sicard, Robitaille, Arbour et Brochu, 2020). Les élèves, surtout les plus jeunes, disent haut et fort ce qui se passe dans leur tête (Madame, je ne vois pas; Madame, est-ce que je peux montrer ma réponse? Madame, est-ce que je peux aller aux toilettes?). Même la meilleure enseignante du monde ne peut arriver à transmettre sa passion et son dynamisme à l'écran, puisque les élèves ne regardent pas juste elle. L'attention des élèves est orientée vers d'autres stimulus. Leur regard est attiré vers les autres élèves et sur ce qu'ils sont en train de faire (changer leur fond d'écran, montrer un jouet, etc.). Au bout du compte, l'enseignement-apprentissage proposé ne peut avoir l'effet escompté, et ce, même avec des stratégies pédagogiques (Messier, 2019) basées sur des données probantes comme l'enseignement explicite (Gauthier, Bissonnette et Richard, 2013; Rosenshine et Stevens, 1986). Pour être capable de gérer une classe en ligne, de façon synchrone, il faut absolument qu'un enseignement explicite des comportements à adopter soit fait en continu (Bissonnette, Gauthier et Castonguay, 2016) comme « attendre son tour de parole, activer le micro lors de son tour de parole, ne pas parler en même temps qu'une autre personne, ne pas faire de bruit inutile, s'assoir correctement, [lever la main], etc. » (Carignan et al., 2020, p. 6).

\section{Un horaire chargé}

Au retour du congé des fêtes 2020-2021, l'enseignement dans les écoles québécoises et ontariennes ${ }^{1}$ s'est fait totalement à distance pendant plusieurs jours, voire plusieurs semaines. Certaines écoles ont opté pour des rencontres synchrones allant d'une demi-heure à plusieurs heures chaque jour; d'autres ont choisi un mélange entre rencontres synchrones et activités à faire en mode asynchrone; d'autres encore ont opté pour $75 \%$ en mode synchrone, par exemple avec Google Meet, et $25 \%$ en mode asynchrone. Dans ce dernier cas de figure, l'école commençait à $8 \mathrm{~h} 45$ et se terminait à $15 \mathrm{~h} 10$ : l'horaire en présentiel a été respecté (tableau 1). Les présences étaient prises au début de la journée et à deux autres moments, car il y avait trois blocs. Ces moments étaient problématiques pour la gestion de classe virtuelle (Carignan et Bissonnette, 2021), car l'enseignant devait constamment rappeler aux enfants de fermer leur micro; c'était la cacophonie.

\footnotetext{
${ }^{1}$ Pour la deuxième année consécutive, l'Ontario a ensuite fermé les écoles d'avril 2021 (et à partir de la mi-mars pour certaines régions) à juin 2021.
} 


\section{Tableau 1}

Exemple d'horaire en $1^{\text {re }}$ année (enseignement virtuel synchrone)

\begin{tabular}{|c|c|}
\hline Heure & Mardi (jour 4) \\
\hline 8 h $45-8$ h 50 & Accueil et présence (début du bloc 1) \\
\hline 8 h $50-9$ h 20 & Français \\
\hline 9 h $20-10$ h 30 & Français \\
\hline 10 h $30-11$ h 05 & $1^{\text {re }}$ pause : temps libre \\
\hline 11 h $05-11$ h 55 & Mathématiques (début du bloc 2) \\
\hline 11 h $55-12$ h 45 & Éducation physique \\
\hline 12 h $45-13$ h 30 & $2^{\mathrm{e}}$ pause $:$ temps libre $\rightarrow$ diner \\
\hline 13 h $30-14$ h 10 & Sciences (début et fin du bloc 3 ) \\
\hline 14 h $10-15$ h 10 & Travail autonome dans Google Classroom \\
\hline $15 \mathrm{~h} 10$ & Fin de la journée \\
\hline
\end{tabular}

En regardant l'horaire, il est possible de constater que le premier bloc dure 105 minutes, soit de $8 \mathrm{~h} 45$ à $10 \mathrm{~h} 30$, sans pause. Les enfants ont alors demandé à leurs parents, à de multiples reprises, quand il y aurait une pause. Lors de la pause, à $10 \mathrm{~h} 30$, c'était le temps de la collation; les enseignants mentionnaient qu'il serait bien que les enfants prennent l'air. Par contre, en plein hiver, avec de jeunes enfants, il est difficile de prendre la collation et d'aller jouer dehors en 35 minutes. Entre la première pause et la deuxième pause, il y a 100 minutes d'enseignement virtuel, devant l'écran. À 14 h 10 , après le court troisième bloc, la grande majorité des enfants étaient en surcharge cognitive (Sweller, 1988). Le travail autonome était donc remis à plus tard dans la journée pour les parents. Après plusieurs heures d'enseignement virtuel synchrone journalier, le travail autonome de $14 \mathrm{~h} 10$ s'est transformé en moment pour prendre l'air. Cet horaire aurait dû mettre davantage de courtes pauses entre les blocs pour permettre aux enfants de bouger un peu et de se changer les idées afin de s'aérer le cerveau.

\section{La conciliation/superposition travail-école}

Dans ce contexte d'école virtuelle en mode synchrone, il est difficile, voire impossible pour les parents, de concilier la vie professionnelle et l'école virtuelle, et ce, particulièrement avec de jeunes enfants au primaire (Carignan et Rodriguez, 2021). Cette conciliation est encore plus difficile quand l'enseignement virtuel adopte un horaire similaire ou presque à celui en présentiel : l'école virtuelle se transforme alors en enseignement à la maison, une modalité de formation, dans ce cas-ci, non choisie par les parents. En effet, les enfants ont constamment besoin des parents : que ce soit pour des problèmes techniques (image saccadée, écran figé, problème de connexion, retour de son, mot de passe qui ne fonctionne pas, écran qui clignote, etc.), pour avoir le matériel nécessaire, pour un câlin ou pour un renforcement positif, par exemple. Plusieurs parents ont dû prendre congé du travail pour pouvoir soutenir adéquatement leurs enfants dans ce double apprentissage : celui du fonctionnement de l'école virtuelle et des apprentissages qui étaient faits pendant les cours.

Les parents ont également dû apprendre les rudiments de Google Classroom, et de tous les autres logiciels et applications utilisés. Le mode de fonctionnement de Google Classroom - qui est une classe virtuelle où un partage de documents est possible - n'est pas nécessairement facile à comprendre pour les parents. Chaque enseignant l'utilise à sa façon et certains enseignants sont plus à l'aise que d'autres. Plusieurs enseignants n'ont jamais reçu la formation nécessaire (ou seulement une formation très courte) et se sont retrouvés, à quelques jours d'avis, à devoir se débrouiller avec cet outil technologique. En outre, pour les parents, il est parfois difficile de trouver les informations sur ce qui est à faire par les enfants pendant le cours et ensuite en devoir. 
D'ailleurs, ce qui émerge le plus de nos observations et de nos échanges entre parents et avec les enseignants est le fait que les parents ont eu de nombreux défis, spécifiquement par rapport aux travaux. En effet, plusieurs parents mentionnent qu'il y a eu beaucoup de devoirs en ligne, trop de ressources et trop de codes différents à gérer, et ceci est encore plus criant lorsque les parents ont plusieurs enfants. Un parent mentionnait que les devoirs avaient été faits et envoyés à l'enseignant, mais celui-ci ne le voyait pas dans Google Classroom. Résultat : le parent a dû faire des photos des écrans, à partir du Chromebook prêté par l'école, pour envoyer les devoirs de son enfant ${ }^{2}$. En bref, plusieurs parents ont dit souhaiter un retour aux outils traditionnels papier-crayon pour éviter que les enfants passent trop de temps devant l'écran et éviter les problèmes techniques liés à la compétence numérique insuffisante des enfants, des parents et de plusieurs enseignants (Karsenti et Grégoire, 2015; Lawrence et Tar, 2018; Chauret, Carignan, Grenon et Collin, 2021). De plus, après une journée d'école virtuelle, les devoirs sont de trop : les enfants sont épuisés et veulent aller jouer pour pouvoir sortir le trop-plein.

\section{Ce qu'en dit la recherche...}

II importe de savoir que de nombreuses recherches sur les effets de l'école virtuelle ont été réalisées aux États-Unis au cours des 20 dernières années, donc bien avant la pandémie. Dans ces études, les chercheurs ont comparé le rendement des élèves recevant un enseignement exclusivement à distance à celui des enfants fréquentant une école traditionnelle de briques et mortier, et recevant un enseignement en présentiel (Bissonnette et Boyer, 2020). Le rapport du Center for Research on Education Outcomes (2015) a comparé le rendement des élèves provenant de 158 écoles à charte virtuelle dans 17 États et le District Columbia avec ceux provenant d'écoles à charte traditionnelle. Les auteurs du rapport ont constaté que les élèves qui fréquentent des écoles virtuelles obtiennent de moins bons résultats en mathématiques et en anglais que ceux qui fréquentent des écoles traditionnelles $(-0,10$ à $-0,39$ écart-type). Plus récemment, Bueno (2020) a analysé le rendement des élèves fréquentant des écoles publiques de la Géorgie de 2007 à 2016 :

la fréquentation d'une école virtuelle à plein temps entraine une réduction statistiquement significative de 0,1 à 0,4 écart-type, en anglais, en mathématiques, en sciences et en sciences sociales pour les élèves du primaire et ceux de première et deuxième secondaire [...] De plus, les résultats de cette étude vont dans le même sens négatif que ceux issus du rapport du Center for Research on Education Outcomes (2015). [...] les élèves qui retournent dans des écoles de brique et de mortier après avoir fréquenté une école virtuelle à plein temps se remettent presque entièrement de la baisse de leurs résultats aux tests $[\ldots]$, le fait de fréquenter une école virtuelle à temps plein est associé à une réduction de $10 \%$ des probabilités d'obtenir un diplôme d'études secondaires (notre traduction, p. 2).

Des résultats similaires ont également été présentés dans l'étude de Fitzpatrick, Berends, Ferrare et Waddington (2020). Ces chercheurs ont analysé les résultats scolaires d'environ 2000 élèves de la $3^{\mathrm{e}}$ à la $8^{\mathrm{e}}$ année sur une période de sept ans, de 2010 à 2017, qui ont fréquenté les écoles en Indiana. L'analyse a montré un effet négatif de la fréquentation d'une école virtuelle en dépit du fait que ces enfants provenaient de milieux plutôt favorisés. En mathématiques, les élèves, qui sont passés d'une école régulière en présentiel à une école virtuelle, ont eu une diminution de leur rendement de $-0,41$ écart-type au cours de la première année suivant le transfert et les effets sont demeurés négatifs la seconde année (-0,48 écart-type) ainsi que la troisième année (-0,50 écart-type). La tendance de l'effet négatif tend à augmenter avec le temps. Le même phénomène se reproduit pour l'apprentissage de la

\footnotetext{
2 Les Chromebook de l'école ne permettent pas aux parents de se connecter à leur compte de courriel ou d'imprimer des documents. Plusieurs sites sont bloqués pour des raisons de sécurité informatique. II n'est pas possible non plus d'envoyer le travail à partir du courriel de l'enfant. 
lecture : il est possible de voir une diminution du rendement au cours de la première année suivant le transfert d'une école en présentiel à une école virtuelle (- 0,29 écart-type) et un maintien des effets négatifs la seconde année (- 0,26 écart-type) est noté avec une accentuation de l'effet négatif la troisième année (- 0,33 écart-type). Comme le soulignent Boyer et Bissonnette (2021) : " ces résultats ont été obtenus auprès d'une population d'élèves provenant de milieux plutôt favorisés ayant un rendement antérieur généralement plutôt élevé. Imaginons les effets de l'école virtuelle sur le rendement des élèves en difficulté et ceux à risques provenant de milieux défavorisés " (p. 12-13). Dans leur recension des écrits, Boyer et Bissonnette (2021) indiquent notamment que : " à la lumière des études et des résultats disponibles, il s'avère inapproprié de recommander une transformation profonde de l'école actuelle au profit d'une école virtuelle offrant uniquement un enseignement à distance » (p. 14).

\section{En guise de conclusion}

La vraie place des enfants et des enseignants est à l'école, en présentiel. Les recherches démontrent notamment que « l'absence d'enseignement en présentiel et de socialisation en classe a un impact négatif sur les résultats des élèves ${ }^{3} »(B u e n o, 2020$, p. 20). Et encore, nous n'avons pas abordé les effets de l'école virtuelle sur les enseignants, leur motivation, leur sentiment d'efficacité personnelle, etc. L'enseignement en ligne constitue une solution d'urgence permettant de garder un contact avec l'école, les enseignants et les camarades de classe, mais n'est clairement pas une solution à long terme. Toutefois, faute de pain, on mange de la galette!

\section{Liste de références}

Aaronson, D., Barrow, L. et Sander, W. (2007). Teachers and student achievement in the Chicago public high schools. Journal of Labor Economics, 25(1), 95-135. http://doi.org/10.1086/508733

Bandura, A. (2013). Auto-efficacité. Le sentiment d'efficacité personnelle. De Boeck Université.

Baudoin, N., Dellisse, S., Lafontaine, D., Coertjens, L., Crépin, F., Baye, A. et Galand, B. (2020). Soutien des enseignants et motivation des élèves durant la pandémie de COVID-19. Formation et profession, 28(4 hors-série), 1-13. https://doi.org/10.18162/fp. 2020.688

Bissonnette, S. et Boyer, C. (2019). Les enfants des milieux socioéconomiques défavorisés sont-ils massivement condamnés à l'échec scolaire? Formation et profession, 27(2), 115-117. https://doi.org/10.18162/fp.2019.a174

Bissonnette, S. et Boyer, C. (2020). Le mirage de l'enseignement à distance. Apprendre et enseigner aujourd'hui, automne, 10-13.

Bissonnette, S., Gauthier, C., et Castonguay, M. (2016). L'enseignement explicite des comportements. Pour une gestion efficace des élèves en classe et dans l'école. Chenelière Éducation.

Boyer, C., et Bissonnette, S. (2021). Les effets du premier confinement, de l'enseignement à distance et de la pandémie de COVID-19 sur le rendement scolaire - Après la pandémie, faudrait-il généraliser l'usage de l'école virtuelle à toutes les clientèles et en toutes circonstances? Montréal : Éditions de l'apprentissage. https://tinyurl.com/f8aszks

Bueno, C. (2020). Bricks and Mortar vs. Computers and Modems: The Impacts of Enrollment in K-12 Virtual Schools. (EdWorkingPaper: 20-250). Récupéré du site du Annenberg Institute de la Brown University. https://doi.org/10.26300/kahb-5v62

Carignan, I., Beaudry, M-C. et Cohene, K. (2020). L'enseignement-apprentissage à la maison en période de pandémie en mettant sa casquette de maman-enseignante-chercheure : un défi de taille. Formation et profession, $28(4$ horssérie), 1-11. http://doi.org/10.18162/fp.2020.689

\footnotetext{
${ }^{3}$ The lack of in-person instruction and classroom socialization negatively impacts students' outcomes.
} 
Carignan, I. et Bissonnette, S. (7 avril 2021). Faire des cours en ligne une option permanente pour les élèves? Jamais de la vie! Journal Le Voyageur, p. 9. https://tinyurl.com/43s4wrkx

Carignan, I. et Rodriguez, J. (24 mars 2021). L'école virtuelle : donner une voix aux parents... et aux parents-enseignants. Journal Le Voyageur, p. 9. https://tinyurl.com/4fz56w9x

Caron, J., Gaudreau, N., Harvey, C., Sicard, S., Robitaille, S., Arbour, M. et Brochu, T. (2020). La gestion de classe au primaire en contexte de pandémie. Formation et profession, 28(4, hors-série), 1-12. https://doi.org/10.18162/fp.2020.674

Center for Research on Education Outcomes. (2015). Online charter school study. Technical report.

Chauret, M., Carignan, I., Grenon, V. et Collin, S. (2021). Les compétences informationnelles des enseignants québécois lors d'une recherche par mots-clés sur un moteur de recherche. Formation et profession, 29(2), 1-18. https://r-libre.teluq.ca/2346/

Fitzpatrick, B. R., Berends, M., Ferrare J. J. et Waddington, R. J. (2020). Virtual Illusion: Comparing Student Achievement and Teacher and Classroom Characteristics in Online and Brick-and-Mortar Charter Schools. Educational Researcher, 49(3),161-175. https://doi.org/10.3102/0013189X20909814

Gauthier, C., Bissonnette, S., et Richard, M. (2013). Enseignement explicite et réussite des élèves. De Boeck.

Hattie, J. (2008). Visible learning: A synthesis of over 800 meta-analyses relating to achievement. Routledge.

Karsenti, T. et Grégoire, P. (2015). Professionnalisation et développement professionnel des enseignants dans un contexte d'intégration des TICE : le cas du Québec. Distances et médiations des savoirs, 11. https://doi.org/10.4000/dms.1140

Lawrence, J. E. et Tar, U. A. (2018). Factors that influence teachers' adoption and integration of ICT in teaching/learning process. Educational Media International, 55(1), 79-105. https://doi.org/10.1080/09523987.2018.1439712

Lee, A. et Gage, N. A. (2020). Updating and expanding systematic reviews and meta-analyses on the effects of schoolwide positive behavior interventions and supports. Psychology in the Schools, 27(5), 783-804. https://doi.org/10.1002/pits.22336

Messier, G. (2019). Le concept de méthode en pédagogie et en didactique : proposition d'un schéma conceptuel. Dans R. Étienne, S. Ragano et L. Talbot (dir.), Peut-on encore parler de méthode pédagogique? 33-49. L'Harmattan.

Rosenshine, B.V., et Stevens, R. (1986). Teaching Functions. Dans M. C. Wittrock (dir). Handbook of Research on Teaching ( $3^{\mathrm{e}}$ éd.). 376-391. Macmillan.

Sweller, J. (1988). Cognitive load during problem solving: Effects on learning. Cognitive Science, 12, 257-285.

Tellier, M. (2020). Socialisation du groupe-classe au temps de la distanciation sociale. Formation et profession, $28(4$ horssérie), 1-15. https://doi.org/10.18162/fp.2020.681

Wiliam, D. (2018). Creating the schools our children need. Learning Sciences International.

Cet article est conforme à la nouvelle orthographe. 